\title{
Engaging Students in the Geosciences using Effective and Versatile Climate and Data Literacy Teaching Modules
}

\author{
ADINA PAYTAN ${ }^{1}$, EMILY WEISS $^{2}$, SARAH PEDEMONTE $^{2}$ \\ AND JUDE APPLE ${ }^{3}$ \\ ${ }^{1}$ University of California, Santa Cruz \\ ${ }^{2}$ LHS, University of California Berkeley \\ ${ }^{3}$ Padilla Bay NERR \\ Presenting Author: apaytan@ucsc.edu
}

Using authentic and locally-relevant environmental data is a valuable teaching tool for providing students with opportunities for self-directed exploration of the natural world. We will present a set of instructional material that focus on climate science and data literacy, prepared by the Lawrence Hall of Science, Berkeley,

CA,

(https://mare.lawrencehallofscience.org/curriculum/climate-dataaclipse-activities). We will demonstrate how these materials can be used in formal (6-12 or university) and informal settings (professional development workshops for teachers and scientists, after school clubs and summer programs). Students engaging in these activities gain a deeper understanding of carbon cycling, and other phenomena related to climate change. The materials guide students through data orientation, interpretation, and synthesis as well as developing and using models and solving problems, constructing explanations, and engaging in argument based on scientific evidence. By solving problems in local contexts, the learners gain knowledge of science content, view science as relevant to their lives and future, and engage in science in socially relevant and transformative ways. Such authentic learning experiences are shown to be an effective approach to improving understanding, confidence, awareness, and interest in geoscience and STEM careers (NRC, 2007). 